\title{
DEFINING THE FRAMEWORK OF ADDRESS SYSTEMS BASED ON STREET-BASED ADDRESS SYSTEM IN KOREA
}

\author{
J. H. Choi ${ }^{1}$, I. H. Park ${ }^{1}$, J. Lee ${ }^{1, *}$ \\ ${ }^{1}$ Dept. of Geoinformatics , University of Seoul, 163 Seoulsiripdaero, Seoul, Korea - \{jihye30, ihpark, jlee $\} @$ uos.ac.kr
}

KEY WORDS: Address, Address system, Components of the address, Street-based address of Korea

\begin{abstract}
:
The address is quasi geospatial data representing the location. There are various ways of address formats, addressing and managing addresses around the world. The Korean address system has been changed from the area-based address system to the street-based address system, which has been implemented since 2014. According to the Ministry of the Interior and Security of Korea, the streetbased address aims at the convenience of directions, rapid response to emergencies, reduction of logistics costs, and strengthening national competitiveness. However, there are limitations in Korea's street-based address system. In this study, we define the components of address systems and analyse the street-based address system of Korea based on the defined elements.
\end{abstract}

\section{INTRODUCTION}

The form, assignment and management of addresses vary from country to country. In each country, the form of the address varies, but the address system used globally can be roughly divided into three types. The first address system is a streetbased addressing system used by many countries such as the United States and the United Kingdom. The second address system is an area-based addressing system, which was used in Korea before it was changed to the street-based address system. The third address system is a hybrid addressing system that combines a street-based addressing system with an area-based addressing system.

As Korea's address system has been changed from the areabased address system to the street-based address system, research on the street-based address system in Korea has been carried out so far. A study has been conducted to suggest ways to improve the standardization of the detailed addresses of street-based address system in Korea (S. W. Hwang Bo, 2017). In addition, a study on geocoding methods and their limitations according to the address system was carried out. Research has also been carried out to complement existing area-based geocoding limitations (J.Lee, 2009). As the street-based geocoding becomes possible through the Korean street-based address system, the research has been carried out to evaluate the location accuracy and to improve it (H. J. No, 2009). In addition, geocoding and reverse geocoding techniques for 3-D addresses were developed using Korean street-based addresses (S. M. Seok, 2016).

The purpose of this study is to define the address components needed for the address system. Also, it is a related study to solve the limitation of street-based address system in Korea.

\section{DEFINITION OF ADDRESS COMPONENTS AND ANALYSIS OF KOREAN STREET-BASED ADDRESS SYSTEM}

\subsection{Definition of address components}

It is necessary to understand the address components before analysing Korea's address system. In order to understand the address system, considerations should be given to address allocation, configuration, and management system aspects. In this study, we define five elements of address as shown in Figure 1 and summarize what each element means.

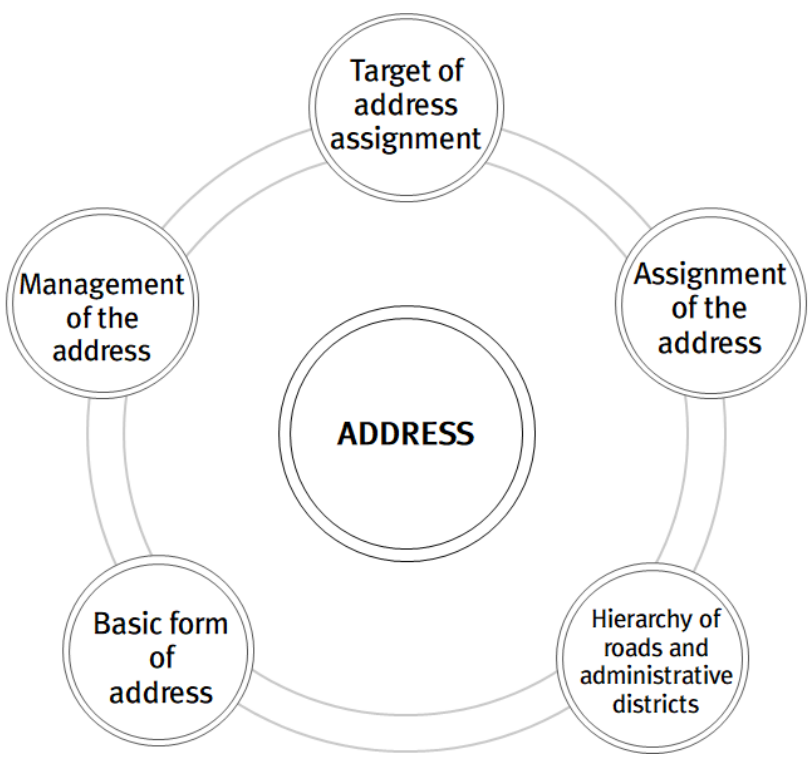

Figure 1. Components of the address

\footnotetext{
* Corresponding author
} 
2.1.1 Target of address assignment: The address is to represent the minimum unit managed by the national or local government agencies in most of countries. In other words, the object to which the address is assigned is the minimum unit managed by the country. When analysing the current addressing targets in world-wise, the process of determining the target of address assignment is roughly divided into two steps as shown in Figure 2. First, a parcel is considered to be a basic unit to assign an address. If there is a building in the parcel, an address is given to the building. However, if there is not a building in the parcel, the parcel will be a target to assign an address. Depending on the building type, the addressing methods and address forms will be different.

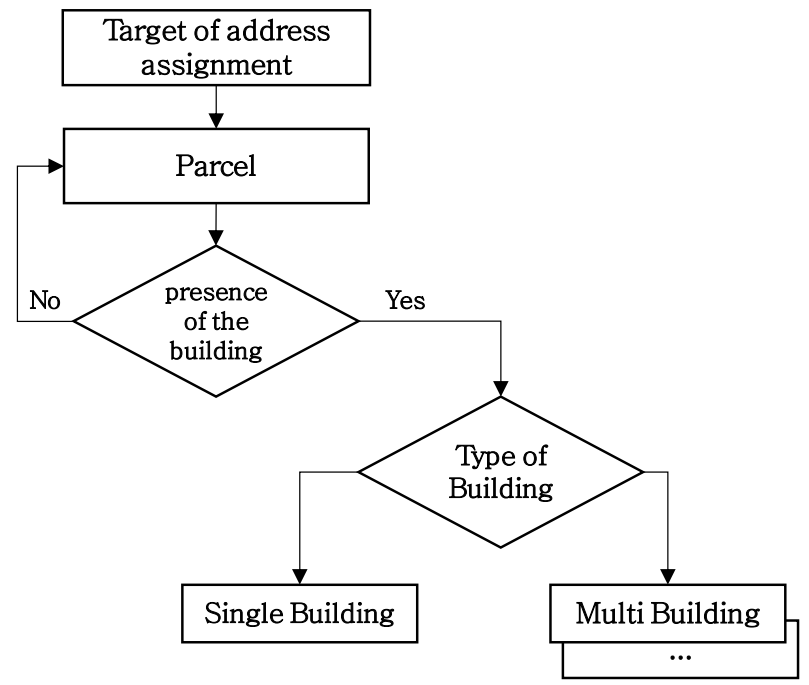

Figure 2. The process of choosing an address assignment target

2.1.2 Assignment of the address: The method of address assignment differs depending on the countries or address systems. Even if the same address system is used, it is common that the addressing methods are not all the same. Since the method of managing addresses varies depending on the method of assigning addresses, this element has a great influence on the address system. In the United States, a street-based address system is used, which divides the road at each intersection and sets the maximum range of address numbers that can be assigned according to the length of road segments. Thereafter, address numbers are assigned to the parcels or buildings in proportion to the length of the road segment.

2.1.3 Basic form of address: The basic form of the address is different depending on the three address systems. A typical example of a street-based address system is the United States, which represents the basic form of an address as 'street number + street + city + state + zip code'. A typical example of the areabased address is Japan, which represents the basic form of an address in the form of 'Zip code + administrative district (a

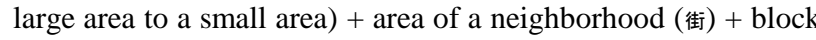
number + building number'. The basic form of an address is efficient in terms of address utilization if the standard is clear and simple. Accordingly, various services can be provided and analysed.
2.1.4 Hierarchy of roads and administration districts: The roads and administration districts used for the address are mostly divided hierarchically. At this time, the hierarchy of roads and administrative districts used for addresses varies from country to country. In Korea, the administrative area used for the address is the same as the actual administrative area, but the US does not match the administrative area used for the address. These layers help people who use the address to understand the space hierarchically.

2.1.5 Management of the address: An address is a representation of where a person's life is based, and the address can be linked to various administrative data and other data about the personal living at that address. If the address is not managed, you can't know which person's data is the record or historical data that has the old address. Therefore, addresses should be managed regardless of the flow of time. Time-series management of new, changed, or deleted addresses is required. In the UK, the address is managed using Postcode Address File and Alias File. Postcode Address File is used to manage the buildings, roads, and town names included in the address and manage the basic address. And it supports the Postcode Address File by managing the changed or newly used address by using Alias File.

\subsection{Analysis of Korean street-based address system}

Based on the five factors defined in the above, we analysed the street-based address system of Korea. First, the address is assigned to the building in Korea. As shown in Figure 3, the building is considered first, and then the address is assigned according to the building type.

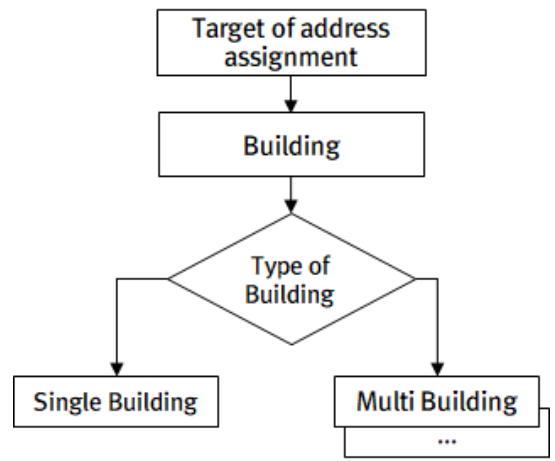

Figure 3. The process of choosing an address assignment target of a street-based address system in Korea

It is impossible to express the entire country as a street-based address system where no building are. Also, since the street name address and the area-based address are mixed, it means the dual address system in Korea.

Another limitation of the Korean street-based address system is to set the road dependency section for roads that are short or unlikely to extend. This means not managing all existing roads individually. Because of this system, a sub-number is created in the building number. This is increases the complexity of the address data. There are two basic types of Korean street-based address: 'Administrative District (small area to large area) + Road name + Building number' and 'Administrative area (small area to large area) Detailed address'. It is difficult to standardize the basic form of the address because the names of the administrative districts vary and the building number mean vary. 
There are 11 types of addresses depending on the hierarchy of administrative districts. And in Korea's street-based address, building numbers refer to five types of buildings. In Korean street-based addresses, the building number refers to a single building, an office building, a multi-house, a multi-building such as an apartment or a school, or an underground building. Therefore, the complexity of the address data increases, and the address is inconsistent. Therefore, it is necessary to make the address data simple and clear. There are administrative districts and road classes in Korea's street-based address system. However, as the level of administrative district in Korea's streetbased address system varies, the basic form of address and address data structure become complicated. Therefore, it is necessary to simplify the administrative level.

As a result of analysing the database of the street-based address provided by the street-based address system of Korea, there is no time series management database of data such as changed street name and address. In other words, time series management of important data is not performed in Korea's street-based address system. Therefore, time series management is necessary for important data management for street-based address system in Korea.

\section{CONCLUSION}

In this study, five elements of address are defined to analyse the street-based address system in Korea. We derived the limit by analysing the Korean street-based address system according to the elements defined in this study. In order to solve the limitations of the street-based address system in Korea, it is necessary to study the reorganization of the street-based address system such as the basic form of the street-based address or the assign method or simplification of address data. In addition, a comprehensive analysis of the address system of countries outside of Korea is required through the components of the five addresses defined in this study.

\section{ACKNOWLEDGEMENTS}

This research was supported by Basic Science Research Program through the National Research Foundation of Korea (NRF) funded by the Ministry of Education (NRF2017R1D1A1B03028890).

\section{REFERENCES}

S. M. Seok., J. Lee., 2016. Development of Geocoding and Reverse Geocoding Method Implemented for Street-based Addresses in Korea, Journal of the Korean Society of Surveying, Geodesy, Photogrammetry and Cartography, 34(1), pp. 33-42.

Lee J., 2009. GIS-based geocoding methods for area-based addresses and 3D addresses in urban areas, Enviroment and Planning B: Planning and Design, Vol.36(1), pp.86-106.

H. J. No., 2009. Assessment and Improvement method of position accuracy of geocoding application for new address system in Korea, Journal of the Korean Cartographic Association, Vol.9(1), pp.63-72.

S. W. Hwang Bo., 2017. A Study on the Development Method of the Detailed Road Name Address, The Korean Society of Cadastre, Vol.33, pp.65-74.
Revised August 2018 